\title{
Target Detection and Recognition of Ground Penetrating Radar using Morphological Image Analysis and Graph Laplacian Regularisation
}

\author{
Jun Dong ${ }^{1}$, Vladimir Stankovic ${ }^{1}$, Nigel Davidson ${ }^{2}$ \\ ${ }^{1}$ Department of Electronic and Electrical, University of Strathclyde, Glasgow \\ ${ }^{2}$ The Defence Science and Technology Laboratory, Kent \\ \{jun.dong, vladimir.stankovic\}@ strath.ac.uk
}

\begin{abstract}
Ground Penetrating Radar (GPR) is often used for detecting non-intrusively buried targets, in road engineering, manufacturing, and in military fields. Based on transmitting high frequency electromagnetic waves, GPR generates high resolution 3D data of the underground structure enabling accurate and fast target detection. However, after inverse Fourier Transform, the 3D GPR images are often out-of-focus and contain high measurement noise. This calls for advanced signal and image processing methods to improve signal-to-noise ratio, isolate the most discriminative features, and perform target detection and localisation. Using a vehicle-mounted GPR array data provided in the 2020 UDRC GPR data challenge, we show that morphological image analysis and semi-supervised learning via graph Laplacian regularisation can detect different types of targets buried at various depths with very low false alarm rate.
\end{abstract}

\section{INTRODUCTION}

Ground Penetrating Radar (GPR) uses high frequency radio waves for detection of the structure of underground objects, based on the difference in electrical properties between the target object and surrounding medium. GPR has been widely used for detection of metallic and non-metallic objects [1] in road engineering, manufacturing, archaeology, and has also become popular for detection of buried targets in military fields, such as land mines and Improvised Explosive Devices (IEDs) [2], [3]. Indeed, the GPR array [2], [3] has shown significant advantages over competing technologies as a non-destructive, remote sensing technique that provides high resolution 3-D data, which helps to make the detection and recognition of targets accurate and fast.

GPR is a non-invasive geophysical technique used for detecting objects underground or analysing the structure in visually opaque materials, based on measuring propagation of emitted high-frequency electromagnetic waves. Though the research and application of GPRs have long history [4], [5], some of the major challenges of using GPR data to detect underground objects are still present. This is due to lowresolution features and out-of-focus GPR images, high measurement noise, especially at large depths, and interference, leading to very low signal to noise ratio (SNR). This calls for advanced signal processing techniques to improve SNR and construct discriminative features.

This work was supported by the Engineering and Physical Sciences Research Council (EPSRC) Grant number EP/S000631/1 and the MOD University Defence Research Collaboration in Signal Processing.
Since a typical output of a GPR system is a spatiotemporal GPR image, various signal and image processing methods can be used for extracting useful information. Traditional popular approaches are based detection of hyperbolas in GPR images, e.g., using Hough transform [6], template matching [7], or Viola-Jones learning algorithm with Haarlike features [8]. However, these approach suffer from false alarms or are complex and require a high number of parameters [2]. In this paper, we propose a low-complexity system, by transforming the time slices of the original dataset acquired by a GPR system into a binary image by performing morphological image analysis to construct distinct features of potential targets, and then classify the constructed features into target/non-target classes using semisupervised learning based on label propagation over graphs. Morphological image processing [9] is a collection of nonlinear operations used to analyse geometrical structures. Morphological operations rely only on the relative ordering of pixel values, and not on their numerical values, and therefore are especially suited to processing binary images.

Classification via Graph Laplacian Regularisation (GLR) has been widely used to classify image and time-series signals, especially when the number of labelled signals that can be used for training is small [10], [11]. In this paper, we use normalised GLR (identified in [12] as the best performing semi-supervised regularisation on graphs classification method for seismic signals), to identify the time slices which contain information about the real targets in order to reduce the false alarm rate and locate the real targets.

In particular, this paper presents a novel post-processing detection method for a stepped frequency continuous wave (SFCW) GPR system. After signal pre-processing, normalisation, and inverse Fourier Transform, the detection of potential targets is performed by carrying out morphological analysis on each time slice. Afterwards, target recognition is performed via GLR-based semi-supervised learning on all potential targets.

The advantage of the proposed method over recent alternatives, such as [2] and [3], is its semi-supervised nature that requires a very small labelled dataset for training (namely, 3.5\% in our simulations), while deep-learning based approaches [2], [3] report results with about two thirds or more of available data used for training. Furthermore, the proposed approach automatically learns system parameters 
from the data and thus, in contrast to prior work [7], [6], does not have many parameters to tune the model. Our simulation results show that the proposed method has a very low false alarm rate. Its low reliance on labelled data, low complexity, and the fact that it can be implemented in real time, make the algorithm a practical solution for future GPR array target detection systems.

This paper is organized as follows: In Section II we describe pre-processing of the GPR data and the method of generating features. In Section III we provide brief background on the morphological image processing and graph signal processing, present the proposed detection and recognition algorithm, including data normalisation and morphology detection method for the constructed features. Section IV presents the simulation results, and Section V contains our conclusions.

\section{DATASET USED AND SIGNAL PRE-PROCESSING}

In this work, we use the data captured by a vehiclemounted SFCW GPR array, which contains 41 transmitreceive channels. The GPR channels were evenly spaced 7.5 $\mathrm{cm}$ apart, covering $3 \mathrm{~m}$ width in total. The GPR system was mounted on a vehicle, driven along test lanes collecting data at $5 \mathrm{~cm}$ intervals. Therefore, a 3D dataset was collected as the GPR array was driven along the lanes. The data was made available as part of a UDRC challenge on automatic target detection for 3D GPR data ${ }^{1}$.

The test lanes included a variety of targets buried at different depths. Multiple test fields were used, and in this paper we focus on a test field that was 100 meters long and shares the same width with the radar antenna array, facilitating for the vehicle to travel through the whole test field and collect a comprehensive dataset. The test field contains 54 targets, randomly located across the whole field.

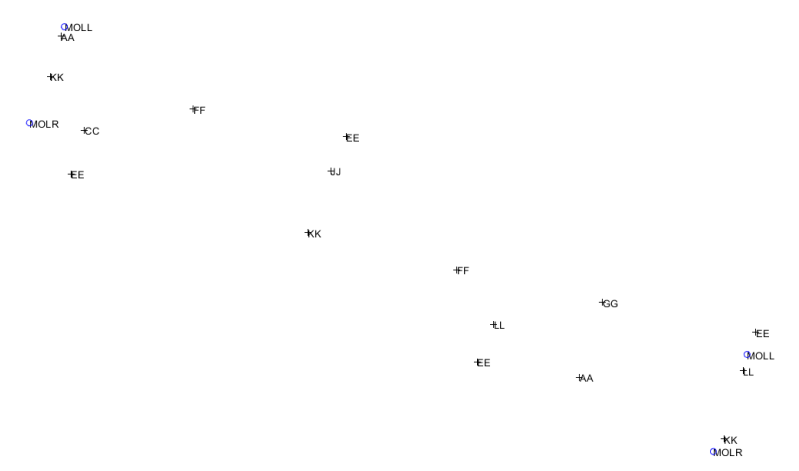

Fig. 1. A part of the plan view of the test lane.

The plan view of the test lane is shown in Fig. 1. The test lane is a rectangular field with 10 landmarks marking its boundary. Blue circles represent the positions of the landmarks of the test lane. The vehicle with the mounted radar was moving within the marked field from one side to another. The cross marks represent the target locations,

${ }^{1}$ https://udrc.eng.ed.ac.uk/sites/udrc.eng.ed.ac.uk/files/attachments/Automatic $\% 20$ Target\%20Detection\%20for\%203D\%20GroundPenetrating\%20Radar\%20Data\%20Challenge.pdf. which are considered as ground-truth target positions. The labels such as 'FF' and 'EE' near the corresponding cross marks indicate the target types; all targets of the same type were buried at a common depth.

As described above, the GPR array collected the data along the test lane at the $5 \mathrm{~cm}$ interval, thus, the data in the original format contains 2000 time slices. To facilitate analysis, we first reshaped the dataset, into a $34 \times 41 \times 2000$ matrix, so that each column represents one radar Transmitter/receiver channel (41 in total). Since the data was collected in the frequency domain due to the characteristic of the SFCW system, to perform analysis in the spatial domain, an inverse fast Fourier transform (IFFT) was carried out along the columns of the dataset, i.e., along the data returned by every array channel, to retain the channel-specific information. Then we process data time slice by time slice, i.e., frame by frame, where each frame is of size $34 \times 41$ pixels.

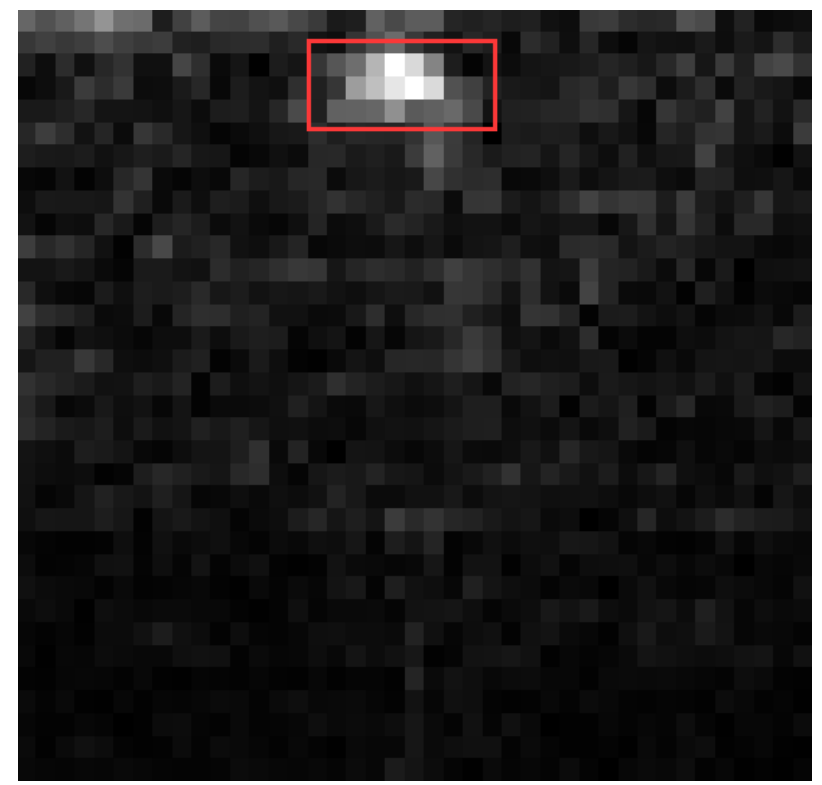

Fig. 2. The magnitude data of the 254th time slice (i.e., frame) of the dataset.

A typical time slice, after IFFT is shown in Fig. 2. Each column corresponds to one channel and the vertical axis shows the depth derived from the propagation time, increasing from the top down. Significantly high values can be observed in the red rectangular box indicating the presence of a target. However, in practice, the targets are often not visible due to measurement noise and clutter. It can also be seen that the targets are pixelated due to the out-offocus problem common to GPR images [4]. Thus, feature construction methods that are robust to noise and blur are required to construct discriminative features necessary for accurate target detection.

\section{Methodology}

This section describes the proposed methodology. In particular, given a 2-D GPR image (e.g., Fig. 2), we use morphological image analysis to construct features and Graph Laplacian Regularisation (GLR)-based label propagation, as advanced semi-supervised classifier. This has been shown to 
perform well when the number of labelled samples is low and dataset is noisy [11], [12].

All matrices will be denoted by capital bold letters, such as $\mathbf{X}$, whose entry in the $i$-th row and $j$-th column will be $x_{i, j}$. Vectors will be denoted by lower-case bold letters, e.g., $\mathrm{x}$, with the $i$-th element being $x_{i}$. Sets are denoted by calligraphic font, e.g., $\mathcal{S}$, and $|\mathcal{S}|$ denotes cardinally of $\mathcal{S}$.

\section{A. Morphological Detection}

As shown in the example in Fig. 2, the targets are characterised by high pixel intensity and the shape. Morphological image analysis is often used to identify the shape or morphology of an object in the image. Thus, in order to extract the shape of the objects, we introduce a Morphological probe [15]. This method requires defining a small shape or template called structuring element, which is slid along the image and compared with the corresponding neighbourhood of pixels [15], [9].

Morphological operations rely only on the relative ordering of the pixel values, and not on their numerical values [15]. Thus, we need to generate a binary image from the original GPR image time slice to extract the features and increase the SNR, which is done using the following equation:

$$
\mathbf{P}=\mathbf{G}-w \cdot \overline{\mathbf{G}},
$$

where, $\mathbf{G}$ is the original 2D time slice data matrix (i.e., the GPR image whose example is shown in Fig. 2$), \overline{\mathbf{G}}$ is its mean, $w$ is a scaling parameter set to 6 in our simulations, and $\mathbf{P}$ is the resulting normalised matrix. Let $p_{i, j}$ be the element (i.e., pixel) of the normalized matrix $\mathbf{P}$ in the $i$-th row and $j$ th column. Then, we feed $p_{i, j}$ into Heaviside step function, that is:

$$
b_{i, j}=H\left(p_{i, j}\right),
$$

where $b_{i, j}$ is the element of the binary matrix, i.e., the morphological detection result, $\mathbf{B}$ in the $i$-th row and $j$-th column.

Based on the target shapes in the training datasets, we experimented with different structuring element shapes and finally adopted a "line" structure element, i.e., 3 pixels in a line as a template. The detection is performed by sliding this 3-pixel line template along the image. The detection result will return a positive detection only if the structuring element "fits" the target, which means if all the 3 pixels in the structuring element calculated by Eq. (2) are larger than 0 . This process would generate a binary image $\mathbf{B}$, as shown in Fig. 3 for the example GPR image from Fig. 2. In B, the pixels with value 1 are identified as potential targets. In the meantime, since the relative location of the time slice and the radar channel is known, the coordinate of potential targets can be calculated. However, as it will be shown in the next section, our simulation results show that this detection result is not reliable enough, as the false alarm rate is very high, that is, due to high measurement noise many false targets are returned. Therefore, a classifier based on GLR is used to increase the accuracy as described next.

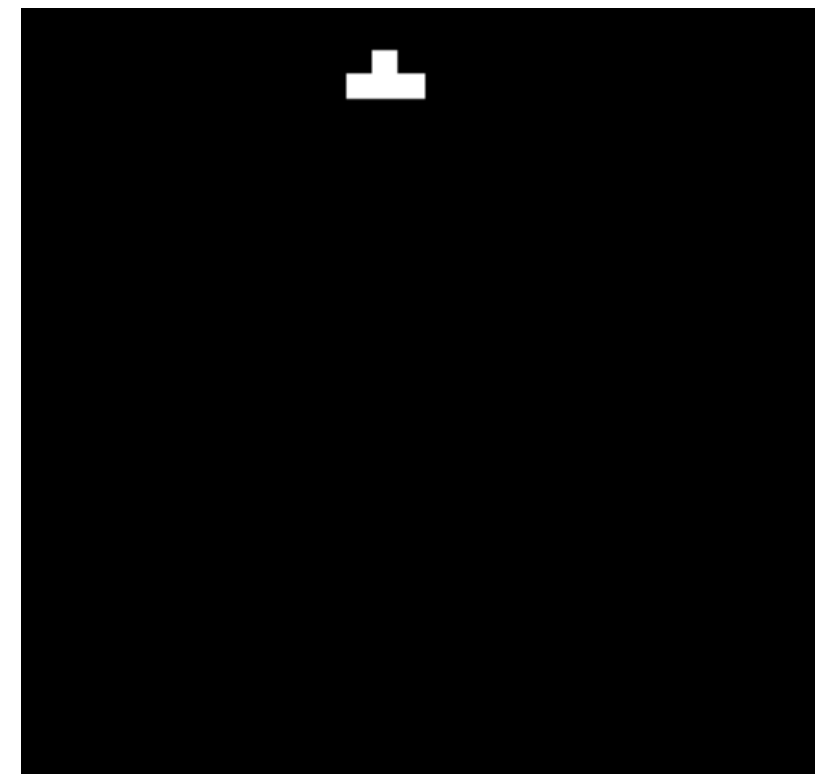

Fig. 3. Morphological detection result for a time slice.

\section{B. GLR-based classification}

The potential targets isolated using morphological image analysis as described in the previous subsection, are fed into a GLR-based classifier.

Semi-supervised learning via label propagation on graphs is widely used for time-series signal classification when the number of labelled signals available for training is very small and hence insufficient to develop good statistical models of the data [10], [11]. The main idea is to represent the classification labels as a graph signal [16] where highly correlated samples are connected with high-weight edges, and then restoring the unknown graph signal samples (corresponding to the test data) using label propagation [10], e.g., via GLR.

Specifically, first, the features extracted from the original time slice data using morphological analysis are embedded into an undirected, connected graph, $\mathcal{G}=(\mathcal{V}, \mathbf{A})$, where $\mathcal{V}$ is the set of vertices and $\mathbf{A}$ is the graph adjacency matrix [16]. Each vertex in $\mathcal{G}, \nu \in \mathcal{V}$ corresponds to one column in the GPR image and is characterised by the corresponding feature vector, e.g., intensity of the corresponding column in the GPR binary image after morphological image processing.

The graph needs to capture well the correlation between the constructed features. Let $f_{k}(i)$ represent the $k$-th feature assigned to Node $\nu_{i}$, that is, the intensity of $k$-th pixel in Column $i$ in the processed GPR image. Then, we set the $(i, j)$ entry in $\mathbf{A}, a_{i, j}$, i.e., the weight of the edge between vertices $i$ and $j$, as:

$$
a_{i, j}=\exp \left\{-\sum_{k=1}^{K} \frac{\left(f_{k}(i)-f_{k}(j)\right)^{2}}{2 \sigma_{k}^{2}}\right\}
$$

where $\sigma_{k}$ represents the graph kernel bandwidth associated to the $k$-th feature, and $K$ is the feature vector dimension, which is the length of the GPR image $\mathbf{B}$ column (in pixels). 
We assign to each vertex, $\nu_{i}$ a discrete graph signal $s_{i}$ that carries the class label of the corresponding event. That is, for Vertex $\nu_{i}$

$s_{i}= \begin{cases}+1, & \text { if } \nu_{i} \text { belongs to Target Class and } i \leq n \\ -1, & \text { if } \nu_{i} \text { does not belong to Target Class and } i \leq n \\ 0, & \text { for } n+1<i \leq N,\end{cases}$

where $n$ and $N$, respectively, represent the number of training samples (i.e., labelled columns) and the total number of image columns in the dataset.

If graph $\mathcal{G}$ captures well the correlation between the constructed features, then the vertices with the same label will be connected by high-weight edges, that is, the graph signal $\mathbf{s}$ will be smooth with respect to $\mathcal{G}$ and we can apply "label propagation" [10], for example, via GLR or normalised GLR, to extrapolate the missing labels (that are initialised to zero in (4)). GLR extrapolates the missing labels by finding the smoothest graph signal under constraints given in the first two lines of Eq. (4) [16].

The combinatorial graph Laplacian matrix is given by $\mathcal{L}=$ $\mathbf{D}-\mathbf{A}$, where $\mathbf{D}$ is the diagonal matrix, given by $d_{i}, i_{i}=$ $\Sigma_{j} a_{i, j}$, and its normalised form is $\mathbf{L}=\mathbf{D}^{(-1 / 2)} \mathcal{L} \mathbf{D}^{(-1 / 2)}$. To perform GLR we define the objective function as:

$$
\begin{aligned}
& \widetilde{\mathbf{s}}=\underset{\mathbf{s} \in R^{N}}{\arg \min }\left(\mathbf{s}^{T} \mathbf{L} \mathbf{s}\right) \\
& \text { s.t. } \widetilde{\mathbf{s}}_{1: n}=\mathbf{s}_{1: n},
\end{aligned}
$$

which has the close-form solution given by [17]:

$$
\widetilde{\mathbf{s}}_{n+1: N}=\mathbf{L}_{n+1: N, n+1: N}^{\dagger}\left(-\mathbf{s}_{1: n}^{T} \mathbf{L}_{1: n, n+1: N}\right)^{T},
$$

where $^{\dagger}$ denotes matrix pseudo-inverse.

\section{RESUlt And Evaluation}

In this section we present and analyse our simulation results. In all our simulations, we use the dataset described in Section II. After detection via morphological image analysis, 1162 potential targets are identified. 40 column vectors with potential targets are used for training the semi-supervised classifier, and the remaining column vectors are used to form the test set.

We evaluate the performance of the proposed system using the probability of detection and probability of false alarm as performance measures. These criteria are widely used in the similar detection problems [18]. We also used the Receiver Operating Characteristic (ROC) curves to evaluate the classifier performance.

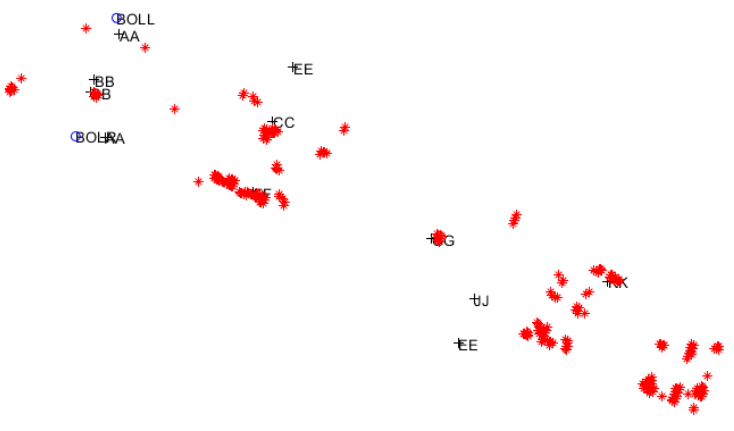

Fig. 4. Detection result before classification.

As part of our study, first we test the performance after morphological analysis only (without semi-supervised learning). The plan view of the test lane showing morphological detection result is shown in Fig. 4. The red asterisks represent the predicted positions and the cross marks represent the actual target locations, i.e., the ground-truth. One can see many correct "hits" (where the red asterisk and the cross marks overlap), but also many false positives (the red asterisk is far from the target real position), and missed targets.

Aiming to reduce the false positive rate, we use semisupervised graph classifier to distinguish the true targets. We use the adjacency matrix as given in Eq. (3) and learn the optimal $\sigma_{k}$ 's from the training data as in [11]. That is, using the training labels, we find the optimal $\sigma_{k}$ 's that maximise the difference between the weights of the edges that connect nodes that belong to the same class and the weights of the edges connecting nodes that belong to the opposite class.

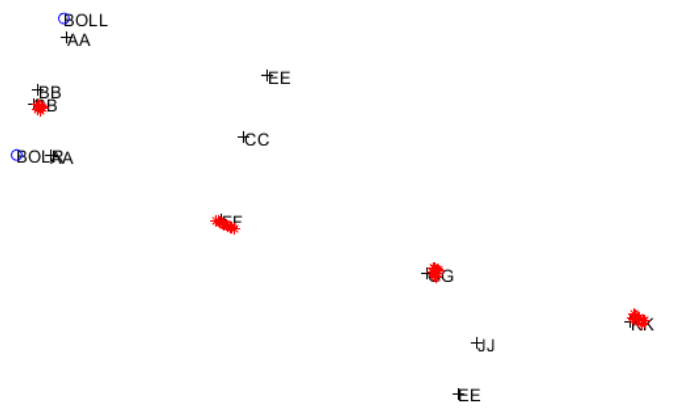

Fig. 5. Detection result after classification.

The plan view of the test lane showing morphological detection result after classification is shown in Fig. 5. Compared to Fig. 4, one can see that most of the false positives are removed while the correct "hits" are kept. High false negative rates are mainly due to very low SNRs in the original dataset, resulting in the absence of prominent features; therefore those targets were unable to be detected by the morphological analysis. Since the semi-supervised classifier takes morphological analysis results as input, it 


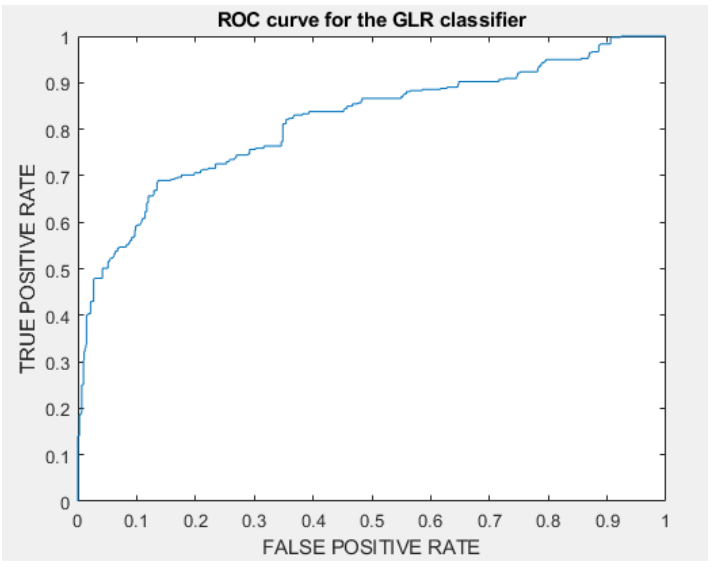

Fig. 6. ROC curve for the proposed GLR classifier.

cannot reduce the false negatives. Note that, these targets are mainly shallow targets, most likely due to low resolution (the out-of-focus problem), high level of interference, and echo of radio waves when they hit the surface [4].

TABLE I

RATING CRITERIA FOR THE TEST

\begin{tabular}{|l|c|c|}
\hline & After classification & Before classification \\
\hline Target detection reports & 144 & 1162 \\
\hline False reports & 20 & 965 \\
\hline Probability of detection & $86.1 \%$ & $17 \%$ \\
\hline Probability of false alarm & $13.9 \%$ & $83 \%$ \\
\hline
\end{tabular}

Table I shows the quantitative performance of the overall proposed system. The results are reported based on the fact that if the predicted position falls within a $0.3 \mathrm{~m}$ radius circle whose center is the real target position, then the predicted result would be counted as true, otherwise it would be counted as false. In Table I, the target detection reports represent how many true positive results have been returned by the classifier. The false report represents the number of positive predictions that are in fact false (false positives). Probability of detection and probability of false alarm show the proportion of true reports and false reports, respectively.

Fig. 6 shows the ROC curve of the GLR classifier for the true target class, which gives the true positive rate against the false positive rate. The larger the area under the ROC curve the better the performance of the classifier.

Semi-supervised classification has significantly reduced the false alarm rate and in turn largely improved the performance. This performance additionally shows the morphological detection and GLR classification method have a satisfactory detection rate and a relatively low false positive rate.

\section{CONCLUSION}

We presented a novel target detection method for data collected by ground penetrating radar. We showed that by performing morphological feature detection and graphbased semi-supervised classification, we can recognise several types of targets buried in the soil, at various depths with a very low false positive rate. Future work will focus on further improving the results, especially for targets at lower depths via de-blurring and further optimising the classifier performance.

\section{ACKNOWLEDGEMENT}

The authors would like to thank Prof. Ian Proudler for proofreading the initial draft of the paper and providing his comments.

\section{REFERENCES}

[1] Daniels, D. J. "Ground penetrating radar," Encyclopedia of $R F$ and microwave engineering. 2004.

[2] M. Pham and S. Lefèvre, "Buried object detection from B-Scan ground penetrating radar data using Faster-RCNN," IGARSS 2018 - 2018 IEEE International Geoscience and Remote Sensing Symposium, 2018, pp. 6804-6807, doi: 10.1109/IGARSS.2018.8517683.

[3] V. Kafedziski, S. Pecov and D. Tanevski, "Detection and classification of land mines from Ground Penetrating Radar data using Faster RCNN," 2018 26th Telecommunications Forum (TELFOR), 2018, pp. 1-4, doi: 10.1109/TELFOR.2018.8612117.

[4] C. Ozdemir, S. Demirci, E. Yiğit, and B. Yilmaz, "A review on migration methods in B-scan ground penetrating radar imaging," Mathematical Problems in Engineering, Hindawi, vol. 2014, 2014.

[5] A. Benedetto, F. Tosti, L.B. Ciampoli, and F. D'amico, Fabrizio, "An overview of ground-penetrating radar signal processing techniques for road inspections," Elsevier Signal Processing, pp. 201-209, vol. 132, 2017.

[6] C. G. Windsor, L. Capineri, and P. Falorni, "A data pair-labeled generalized Hough transform for radar location of buried objects," IEEE Geosci. Remote Sens. Lett., vol. 11, pp. 124-127, 2014.

[7] G. Terrasse, J.-M. Nicolas, E. Trouve, and E. Drouet, "Automatic localization of gas pipes from gpr imagery," in EUSIPCO-2016, pp. 2395-2399, 2016.

[8] C. Maas and J. Schmalzl, "Using pattern recognition to automatically localize reflection hyperbolas in data from ground penetrating radar," Computers \& Geosciences, vol. 58, pp. 116-125, 2013.

[9] P. Soille, Morphological Image Analysis: Principles and Applications, Springer Science \& Business Media, 2013.

[10] M. Belkin and P. Niyogi, "Using manifold structure for partially labeled classification", NeurIPS, 2003.

[11] M. Ye, V. Stankovic, L. Stankovic, and G. Cheung, "Robust deep graph based learning for binary classification," IEEE Trans. Signal Inf. Process. Networks, Nov. 2020.

[12] J. Li, L. Stankovic, S. Pytharouli, and V. Stankovic, "Automated platform for microseismic signal analysis: Denoising, detection and classification in slope stability studies", IEEE Trans. Remote Sensing, to appear, 2021.

[13] G. Cardone, G. Cincotti, P. Gori, and M. Pappalardo, "Optimization of wide-band linear arrays," IEEE Trans. Ultrasonics, Ferroelectrics, and Frequency Control, vol. 48, no. 4, pp. 943-952, July 2001.

[14] H. L. Van Trees, Detection, Estimation and Modulation Theory: Optimum Array Processing. New York: Wiley, 2002.

[15] "Morphological Image Processing", Cs.auckland.ac.nz, 2021. [Online]. Available: https://www.cs.auckland.ac.nz/courses/compsci773s1c/lectures/ImageProcessinghtml/topic4.htm. [Accessed: 30- Apr- 2021].

[16] A. Ortega, P. Frossard, J. Kovacevic, J. M. F. Moura and P. Vandergheynst, "Graph Signal Processing: Overview, challenges, and applications," in Proceedings of the IEEE, vol. 106, no. 5, pp. 808-828, May 2018.

[17] S. Boyd and P. Vandenberghe, Convex Optimization, Cambridge University Press, 2004.

[18] X. Shi, D. Cheng, Z. Song and C. Wang, "A real-time method for landmine detection using vehicle array GPR," 2018 17th International Conference on Ground Penetrating Radar (GPR), 2018, pp. 1-4, doi: 10.1109/ICGPR.2018.8441584. 